\title{
Variation in free and bound water molecules in different homeopathic potencies as revealed by their Fourier Transform Infrared Spectroscopy (FTIR)
}

\author{
Indrani Chakraborty 1,3, Soumita Datta ${ }^{3}$, Anirban Sukul ${ }^{3,4}$, \\ Rathin Chakravarty ${ }^{3}$, Nirmal C. Sukul ${ }^{2,3,4}$
}

\author{
${ }^{1}$ Department of Zoology, Vidyasagar College for Women, Kolkata, West Bengal, India \\ 2 Department of Zoology, Visva-Bharati, Santiniketan, West Bengal, India \\ ${ }^{3}$ Molecular Homeopathy Research Unit, Kolkata, West Bengal, India \\ ${ }^{4}$ Sukul Institute of Homeopathic Research, Santiniketan, West Bengal, India
}

\begin{abstract}
Homeopathic potencies $12 \mathrm{cH}$ and above cross the Avogadro number, and as such do not contain any original drug molecules in their aqueous ethanol medium. It is thought $\mathrm{H}$-bonded water structures preserved by ethanol carry the information of initial drug molecules. Potentized drugs show some differences with respect to their infrared (IR) absorption spectra. In a water-ethanol solution, free water molecules vary according to the concentration of ethanol. In the present study the concentration of ethanol has been kept constant at 0.03 molar fractions in 6 different homeopathic potencies. Aim: to see whether different homeopathic potencies having fixed ethanol content show variation in FTIR spectra and also free water molecules. Two potencies like $8 \mathrm{cH}$ and $32 \mathrm{cH}$ of three homeopathic drugs Natrum mur, Cantharis and Nux vomica were used in the study, and their ethanol concentration was kept fixed at 0.03 molar fraction. The control was considered to be aqueous ethanol at the same concentration. Spectrum of reference water was also taken. Fourier transform infrared (FTIR) absorption spectra were obtained in the wave number region of $4000-2800 \mathrm{~cm}^{-1}$. The half-width at half-maximum was measured for each spectrum. The intensity of each spectrum was normalized at $3410 \mathrm{~cm}^{-1}$ close to the peak. The difference spectrum (absorbance of drug solution - absorbance of reference water) for each drug and the control was obtained. FTIR spectra showed variation in absorbance intensity on both the high and low frequency side of the $\mathrm{O}-\mathrm{H}$ stretching band in different drugs as well as the control. The C-H stretching band of $2977 \mathrm{~cm}^{-1}$ also showed variation in intensity in different drugs. In the difference spectra the absorbance intensity at the dip at $3630 \mathrm{~cm}^{-1}$ varied in different drugs and the control. The decrease in intensity at $3630 \mathrm{~cm}^{-1}$ and subsequent rise in intensity at lower frequency region represent the level of free water molecules and strong alcoholic $\mathrm{O}-\mathrm{H}$ band around $3250 \mathrm{~cm}^{-1}$, respectively. The drug and the control solutions show distinct variation in their FTIR spectra. The drugs have different levels of bound and free water molecules although their ethanol concentration is same.
\end{abstract}

Keywords: Homeopathic potencies, FTIR spectra, free water molecules, ultra high dilution. 


\section{Introduction}

Potentized homeopathic drugs of the $12^{\text {th }}$ potency have a dilution $10^{24}$ which crosses the Avogadro number. For this, all homeopathic potencies from $12^{\text {th }}$ onwards are not expected to contain any original drug molecules from which they have been prepared. However, there are many clinical as well as experimental evidences which show that the potencies produce specific biological effects $[1,2,3]$. Homeopathic potencies are prepared in aqueous ethanol, and usual chemical analysis shows that all of them contain ethanol and water. In our earlier studies we have observed that homeopathic potencies show some differences from each other and from their diluent medium, aqueous ethanol, by their infrared spectra $[4,5]$.

Infrared absorption measures the vibrational frequency of bonds .One theory explaining the physical basis of a homeopathic potency states that hydrogen-bonded water structures preserved by ethanol molecules constitute the physical basis of a potency $[2,3]$. These water structures vary in different potencies of a drug, and also in different potentized drugs. Recent experimental evidences show that water carries the information of a homeopathic potency [6, 7, 8]. The purpose of the present study is to see whether Fourier Transform Infrared (FTIR) spectra show any difference in different potencies of a drug, different potentized drug, and free water molecules.

A survey of the existing literature on the FTIR spectra of aqueous ethanol shows that the spectra vary according to the concentration of ethanol in the water-ethanol mixture [9, 10,11]. In our study we have kept the concentration of ethanol same, 0.03 molar fraction, in all the test solutions studied. We can, therefore, anticipate theoretically that the FTIR spectra of all the test solutions should be more or less same. Since water structures are thought to be the main factor in a potency we have obtained a difference spectra (absorbance of test solution - absorbance of reference water) of drug solutions in aquous ethanol with a view to determining the role of water in the drug solutions.

\section{Material and Methods}

Homeopathic drugs used in this study were Natrum mur $6 \mathrm{cH}, 30 \mathrm{cH}$, Cantharis $6 \mathrm{cH}, 30 \mathrm{cH}$ and Nux Vomica $6 \mathrm{cH}, 30 \mathrm{cH}$, produced by Dr. Reckeweg, Germany. Sealed vials of the drugs were purchased from the local market, Kolkata. All the drugs were in $90 \%$ ethanol as mentioned in their labels. The control $90 \%$ ethanol was prepared in the laboratory from dehydrated ethanol obtained from Bengal Chemicals and Pharmaceuticals Ltd, Kolkata. In order to equilibrate the alcohol content of the drugs with our $90 \%$ alcohol we further enhanced each potency by 2 through the usual process of dilution with our alcohol 1:100 followed by succussion. Thus $6^{\text {th }}$ or $30^{\text {th }}$ potency of each drug was diluted with our $90 \%$ ethanol 1:100 and then manually succussed 10 times over a book to make it $7^{\text {th }}$ or $31^{\text {st }}$ potency. Each of this potency was then diluted with our ethanol 1:100 and succussed 10 times to make it $8^{\text {th }}$ of or $32^{\text {nd }}$ potency. In this way each drug was equilibrated with our control solution with respect to their alcohol content. By adding appropriate volume of sterile distilled water to each drug and the control the alcohol content was further reduced to 0.03 molar fraction. Pure HPLC water was obtained from our local chemical supplier, Kolkata. Technical data for this water shows that it has $0.0005 \%$ nonvolatile matter, $0.000001 \%$ chloride, $0.00001 \%$ sulphate, maximum UV absorption 0.005 in the wavelength range of $250-400 \mathrm{~nm}$ and 0.01in the range of 205-210 nm.

The IR absorption spectra were recorded by a FTIR spectrometer (Perkin Elmer, Spectrum 100) at $22^{\circ} \mathrm{C}$ and relative humidity $65 \%$ in nitrogen gas atmosphere using ATR (attenuated total reflection mode). The resolution was $2 \mathrm{~cm}^{-1}$. A drop of the drug, the control solution or reference water was put in between the plates and covered with a lid. Control solution means 0.03 molar fraction of ethanol, not prepared by dynamization. We also used dehydrated ethanol (99.9\%). All measurements were taken in the wave number region of $4000-2800 \mathrm{~cm}^{-1}$. We mainly focused our attention on the $\mathrm{OH}$ stretching vibration at $2800-3800$ 
$\mathrm{cm}^{-1}$, but also observed C-H stretching band at $2977 \mathrm{~cm}^{-1}$. The half-width at half-maximum (HWHM) was measured for each spectrum. The intensity of each absorption spectrum was normalized at $3410 \mathrm{~cm}^{-1}$ close to the peak position. The difference spectra for each drug and the control (ethanol only 0.03 molar fraction) solution was obtained by subtracting the intensity normalized reference water spectrum from each drug or the control solution. This would help in understanding the role of water in the water-alcohol solution of each drug and the control.

\section{Results}

The FTIR spectra of the two potencies of each drug, control and reference water are presented in Figures 1 3. In all cases water showed the highest absorbance intensity. Further, the $8^{\text {th }}$ potency had always the higher intensity than the $32^{\text {nd }}$ potency in all the three drugs (figures $1,2,3$ ). While the control solution (ethanol 0.03 molar) mostly occupied the second highest position next to water with respect to intensity, Natrum mur $8 \mathrm{cH}$ occupied this position (figure 3). The peak position in water, control and drug solutions was almost same at $3284 \mathrm{~cm}^{-1}$. The peak position of dehydrated ethanol shows a blue shift. The $\mathrm{C}-\mathrm{H}$ stretching vibration band was at $2977 \mathrm{~cm}^{-1}$ and its absorbance intensity varied in different drugs (figures 1,2,3). However, in case of dehydrated ethanol the intensity of $\mathrm{CH}$ stretching band almost equaled that of the $\mathrm{OH}$ stretching one.

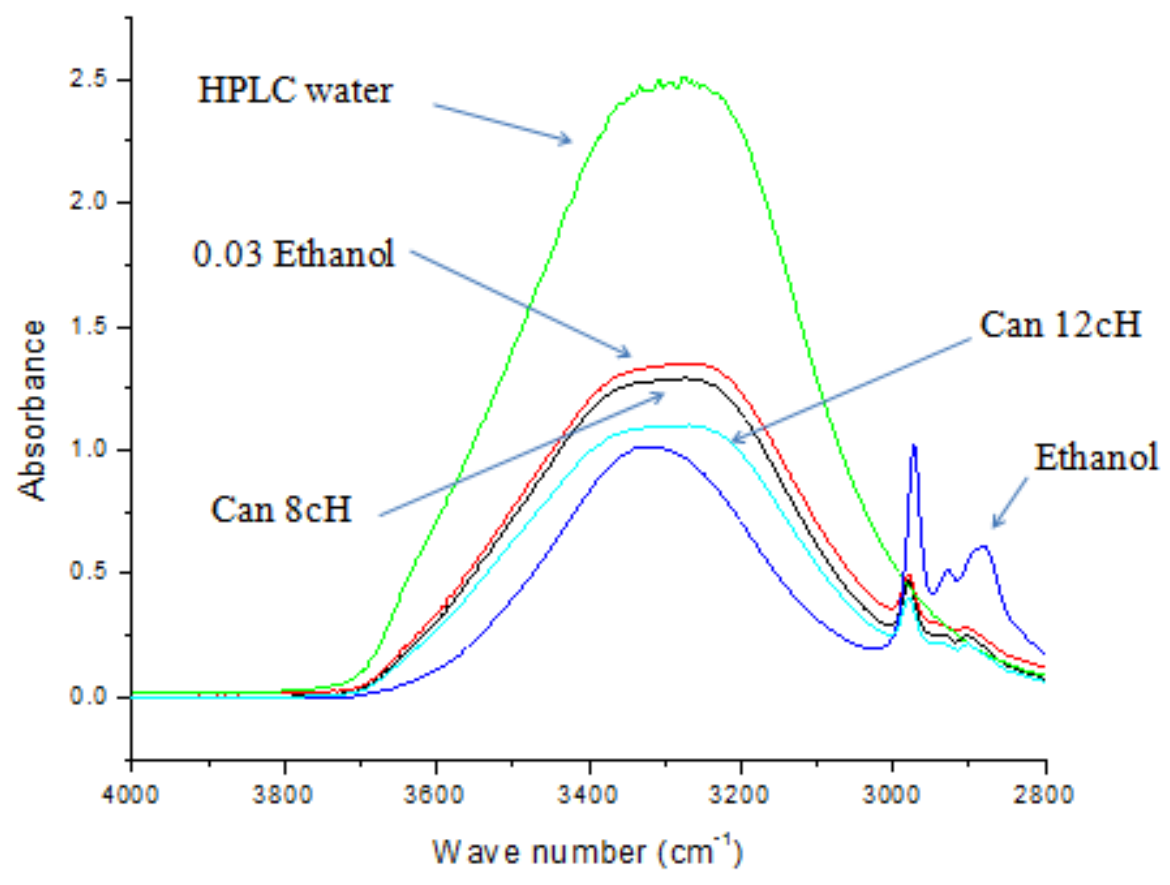

Figure 1: FTIR spectra of Cantharis $8 \mathrm{cH}$ and $32 \mathrm{cH}$, water, 0.03 molar ethanol (control) and dehydrated ethanol. The drugs and the control solutions were in 0.03 molar fraction of ethanol. 


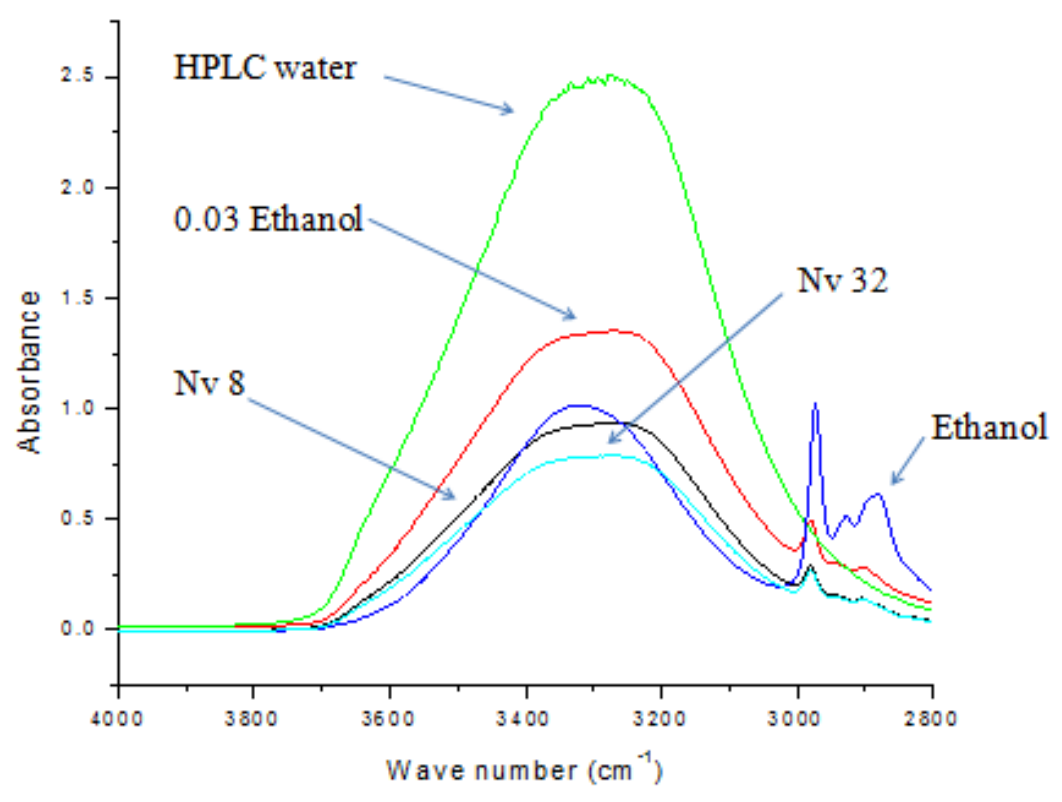

Figure 2: FTIR spectra of Nux vomica $8 \mathrm{cH}$ and $32 \mathrm{cH}$, water, 0.03 molar ethanol (control) and dehydrated ethanol. The drugs and the control solutions were in 0.03 molar fraction of ethanol.

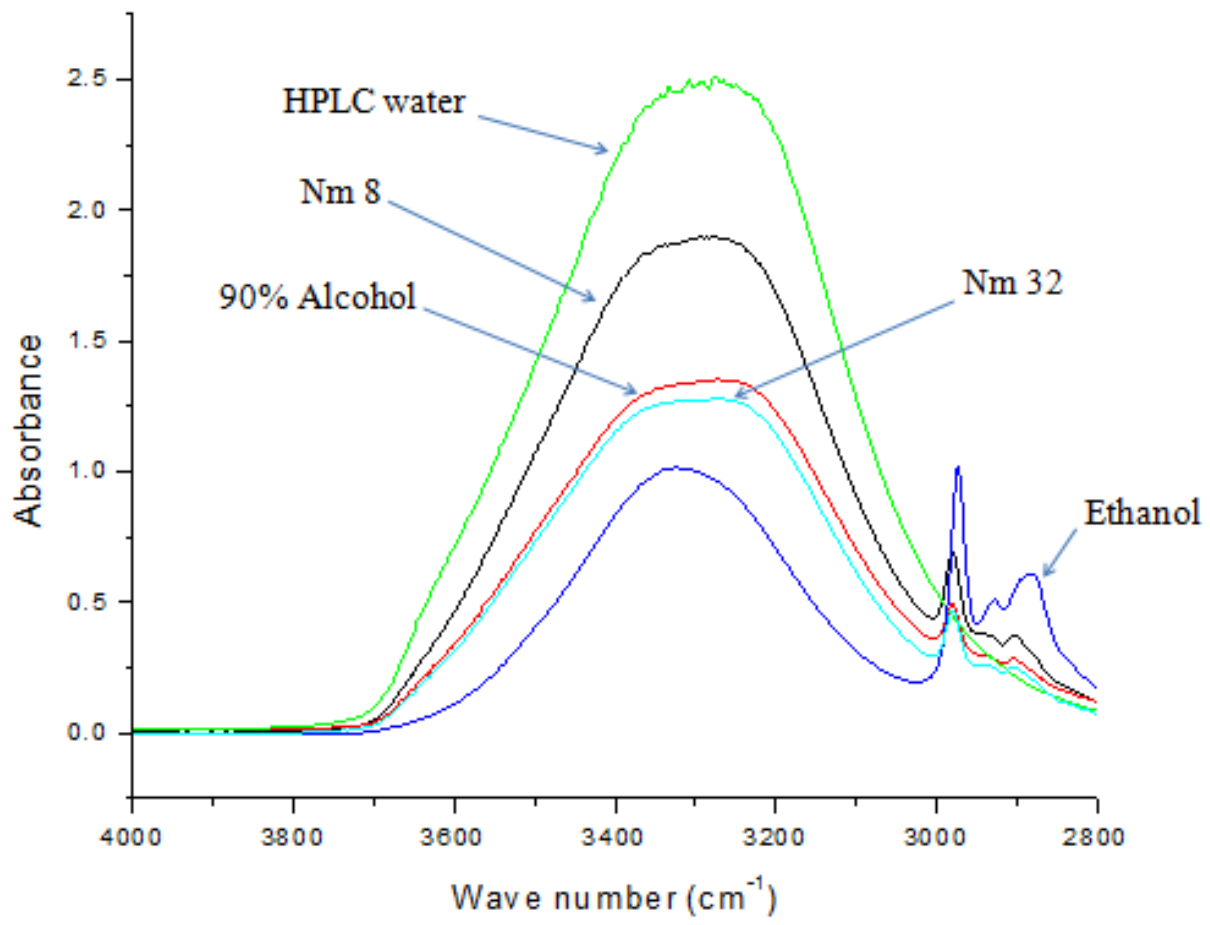

Figure 3: FTIR spectra of Natrum mur $8 \mathrm{cH}$ and $32 \mathrm{cH}$, water, 0.03 molar ethanol (control) and dehydrated ethanol. The drugs and the control solutions were in 0.03 molar fraction of ethanol. 
The half width at half maximum (HWHM) in the high frequency side was narrower by $4 \mathrm{~cm}^{-1}$ in $N a t r u m ~ m u r$ $32 \mathrm{cH}$, Cantharis $8 \mathrm{cH}$ and Cantharis $32 \mathrm{cH}$ relative to water (Table 1). HWHM was narrower by $22 \mathrm{~cm}^{-1}$ relative to water in case of $N u x$ Vom $8 \mathrm{cH}$. In all other cases the HWHM was same as that of water (table 1).

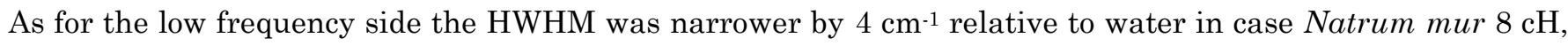
$32 \mathrm{cH}$ and Nux Vom $32 \mathrm{cH}$ (Table 1). The HWHM was narrower relative to water by $11 \mathrm{~cm}^{-1}$ in case Cantharis $8 \mathrm{cH}, 32 \mathrm{cH}$ and by $8 \mathrm{~cm}^{-1}$ in case of $N u x \operatorname{Vom} 8 \mathrm{cH}$. It is wider relative to water by $4 \mathrm{~cm}^{-1}$ in case of the ethanol solution (Table 1).

Table 1.: Data on the half width at half maximum (HWHM) in the high and low frequency side of the OH stretching vibration at $3284 \mathrm{~cm}^{-1}$ in homeopathic drugs and their diluent medium 0.03 molar ethanol relative to water. All the drugs and their medium have been equilibrated at 0.03 molar fraction of ethanol.

\begin{tabular}{|l|l|l|}
\hline Drugs/Medium & $\begin{array}{l}\text { HWHM in the high frequency side } \\
\text { relative to water }\end{array}$ & $\begin{array}{l}\text { HWHM in the low frequency side } \\
\text { relative to water }\end{array}$ \\
\hline Water & - & - \\
\hline 0.03 molar ethanol (medium) & Same & Wider by $4 \mathrm{~cm}^{-1}$ \\
\hline Natrum mur $8 \mathrm{cH}$ & Same & Narrower by $4 \mathrm{~cm}^{-1}$ \\
\hline Natrum mur $32 \mathrm{cH}$ & Narrower by $4 \mathrm{~cm}^{-1}$ & Narrower by $4 \mathrm{~cm}^{-1}$ \\
\hline Cantharis $8 \mathrm{cH}$ & Narrower by $4 \mathrm{~cm}^{-1}$ & Narrower by $11 \mathrm{~cm}^{-1}$ \\
\hline Cantharis $32 \mathrm{cH}$ & Narrower by $4 \mathrm{~cm}^{-1}$ & Narrower by $11 \mathrm{~cm}^{-1}$ \\
\hline Nux vom $8 \mathrm{cH}$ & Narrower by $22 \mathrm{~cm}^{-1}$ & Narrower by $8 \mathrm{~cm}^{-1}$ \\
\hline Nux vom $32 \mathrm{cH}$ & Same & Narrower by $4 \mathrm{~cm}^{-1}$ \\
\hline
\end{tabular}

The difference spectra (absorbance of drug solution - absorbance of reference water) in the wave number region of $4000 \mathrm{~cm}^{-1}-2800 \mathrm{~cm}^{-1}$ were shown in Figure 4 . The absorbance intensity at the dip at $3630 \mathrm{~cm}^{-1}$ varied in different drug solutions and the diluent medium (ethanol 0.03 molar fraction from 0.03 to 0.08 ) (figure 4). The absorbance at the peaks in the lower frequency region $3440-3255 \mathrm{~cm}^{-1}$ varied in different drugs and the diluent medium from 0.01 to 0.16 (figure 4). The shape of the spectra of the test solutions also showed marked variation from each other (figure 4). 


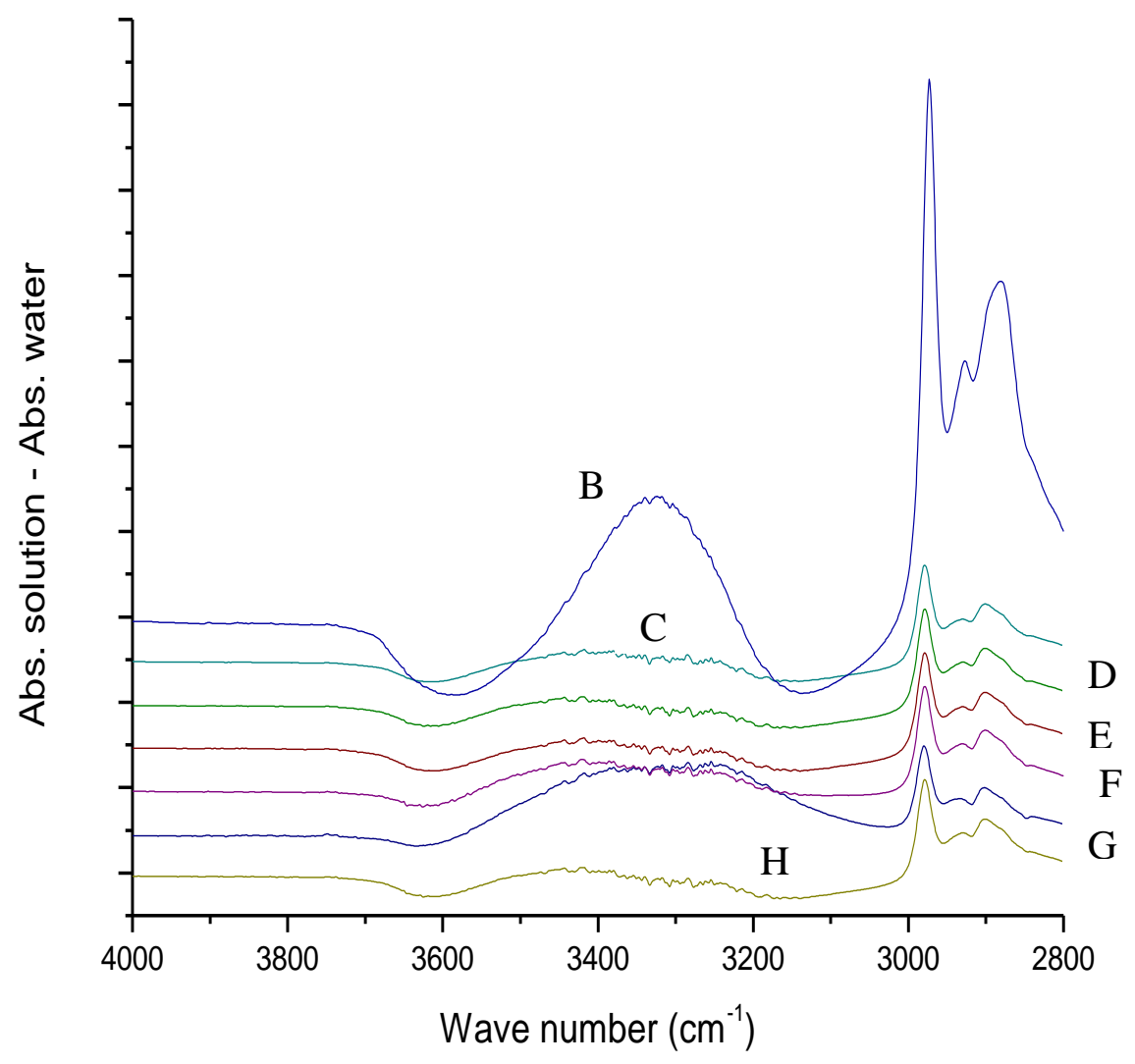

Figure 4: Difference spectra (absorbance of solution at 0.03 molar fraction - absorbance of reference water). The ethanol solution spectra and the water spectrum were intensity normalized at $3410 \mathrm{~cm}^{-1}$. The sharp peaks at $2977 \mathrm{~cm}^{-1}$ represent $\mathrm{C}-\mathrm{H}$ stretching vibration and bands of the ethyl group of ethanol. The vertical axis represents difference in absorbance after intensity normalization.
B: Abs 0.03 ethanol (control) - Abs water
C: Abs Nm 8 - Abs water
D: Abs Nm $32-$ Abs water
E: Abs Can 8-Abs water
F: Abs Can $32-$ Abs water
G: Abs Nv $8-$ Abs water

H: Abs Nv $32-$ Abs water

\section{Discussion}

Pure water forms tetrahedrally coordinated structures [12]. Ethanol with one hydroxyl group $\left(\mathrm{CH}_{3} \mathrm{CH}_{2} \mathrm{OH}\right)$ is characterized by hydrogen bonding patterns which may be regarded as linear chains [13]. In the mixture of water and ethanol each component tends to maintain to some extent its individual $\mathrm{H}$-bonded patterns. However, H-bonded network in aqueous ethanol undergoes a significant rearrangement which influences the structure and dynamics of the two components with respect to those of the pure liquids [12, 14]. Essential structural rearrangement occurs in water-ethanol solutions. Ethanol concentrations in the range of $15-20 \%$ $\mathrm{w} / \mathrm{w}$ show stronger hydrogen bonding as compared to pure water. IR absorption measurements of aqueous ethanol show that ethanol molecules are monomolecularly dispersed and surrounded by water molecules at low alcohol concentration. At higher concentrations water molecules are too few to accommodate all ethanol 
molecules in the clathrate-like structure [11]. Water ethanol mixtures form heterogenous micellar microaggregates as evidenced by other studies like sound absorption, X-ray scattering and light scattering $[15,16]$.

Reference [10] reported that the HWHM in the high frequency region was narrower by $6 \mathrm{~cm}^{-1}$ than that of reference water for 3\% ethanol (0.03) solution, but the HWHM in the low frequency region remained unaltered. This was attributed to the change in the shape of spectra in aqueous ethanol. Using computer analysis of vibrational spectra of water ethanol solutions Burikov et al. (2012) reported dependence of the major spectral components in the $\mathrm{OH}$ stretching vibration band on the ethanol concentration in water [17]. In our study we observed a marked variation in the HWHM in different potentized drugs on both high and low frequency regions although the ethanol concentration was same (0.03) with all the test solutions (table 1). This shows that different drugs have different shape of spectra, and this may be due to the difference in the H-bonded network in the drug solutions. The authors [10] further observed in the difference spectra (absorbance of aqueous ethanol solutions - absorbance of reference water) that the deepest dip in intensity in the high frequency region of $3600 \mathrm{~cm}^{-1}$ and subsequent rise in the low frequency varied exactly according to the concentrations of alcohol $(0.01,0.02,0.03)$. In our study we observed a marked variation in intensity decrease at $3600 \mathrm{~cm}^{-1}$ and increase in intensity in the low frequency reign $3441-3256 \mathrm{~cm}^{-1}$ in different potentized drugs (figure 4). However, the alcohol content was same (0.03) in all the test solutions. Reference [10] attributed this fall and rise in intensity to the level of free water molecules and stronger $\mathrm{O}-\mathrm{H}$ bond around $3250 \mathrm{~cm}^{-1}$, respectively. The deeper was the dip at $3600 \mathrm{~cm}^{-1}$, the lesser was the free water molecules due to addition of alcohol. In our study 0.03 molar ethanol (control) showed the deepest deep at $3600 \mathrm{~cm}^{-1}$. This means the control has the least free water molecules. It also shows strongest $\mathrm{OH}$ bond around $3250 \mathrm{~cm}^{-1}$ as compared to the potentized drugs studied. Besides Nuxvom $8 \mathrm{cH}$, other potentized drugs have weaker $\mathrm{OH}$ bond around $3250 \mathrm{~cm}^{-1}$ (Figure 4). Potentized drugs also have greater number of free water molecules. Our study shows that different potentized drugs have different amount of free water molecules and consequently different level of bound water molecules. It also shows that alcoholic $\mathrm{O}-\mathrm{H}$ bond strength also varies in different drugs (figure 4). This may be due to the difference in the H-bonded network in different drugs and also their bond strength induced by successive dilution and succussion during the preparation of homeopathic potencies [2]. This FTIR study shows that water molecules, free and bound, contribute significantly to the variation in homeopathic potencies.

\section{Acknowledgement}

We thank the Director, Bose Institute, Kolkata for providing instrumentation facilities. We thank Mr. Pranab De, Department of Chemistry, Bose Institute for his help in obtaining the FTIR spectra of our samples. A special word of thanks to Prof. K.P. Das, Department of Chemistry, Bose Institute for critically reviewing this paper. We also thank the Bholanath Chakravarty Memorial Trust for their constant cooperation and inspiration for this research work.

\section{References}

[1] Cuckerat M, Haugh MC, Gooch M, Boissel JP. Evidences of clinical efficacy of homeopathy: A meta analysis of clinical trials, Eur J Clin Pharmacol. 2000; 56: 27-33.

[2] Sukul NC, Sukul A. High Dilution Effects: Physical and Biochemical Basin, Kluwer Academic Press, Dordrecht. 2004: 130.

[3] Sukul NC, Sukul A. Molecular mechanism of action of homeopathic potencies with reference to holism, Environ Ecol. 2009; 27: 71-77. 
[4] Sukul NC, De A, Dutta (Nag) R, et al. Nux Vomica 30 prepared with and without succession shows antialcoholic effect on toads and distinctive molecular association, Br. Hom J. 2001; 90: 79-85.

[5] Sukul NC, Ghosh S, Sukul A, Sinhababu SP. Variation in Fourier transform infrared spectra of some homeopathic potencies and their diluent media, J Alt Com Med. 2005; 11: 807-812.

[6] Mondal S, Sukul (nee Chunary) S, Sukul NC. Water as carrier of information of heat shock and drug effect between two groups of Adhatoda Vasica plants, Int J High Dilution Res. 2012a; 11: 60-68.

[7] Mondal S, Sukul S, Sukul NC. Transfer of effect of heat shock and drug treatment from one plant to another through water, J Altern Med Res. 2012b; 4: 179-185.

[8] Chakraborty I, Sukul NC, Sukul A, Chakravarty R. Transfer of the anti-alcoholic effect of Nux Vomica $200 \mathrm{CH}$ through water from one group of toads to another under alcohol anesthesia, Int J High Dilution Res. 2012; 11: 216-223.

[9] Mizuno K, Miyashita Y, Shindo Y. NMR and FTIR studies of hydrogen bonds in ethanol-water mixtures, J Phys Chem. 1995; 99: 3225-3228.

[10] Nishi N, Takamuku T, Yamaguchi T. Hydrogen bonding cluster formation and hydrophobic solute association in aqueous solution of ethanol, J Phys Chem. 1995; 99: 462-468.

[11] Burikov S, Dolenko T, Patsaeva S, Starokura Y, Yuzhakov V. Raman and IR spectroscopy research on hydrogen bonding in water-ethanol systems, Molecular Physics. 2010; 108(18): 2427-2436.

[12] Skaf MS, Ladanyi BM. Molecular dynamics simulation of the wave vector-dependent static dielectric properties of methanol water mixtures, J Chem Phys.1995; 102: 6542-6551.

[13] Saiz L, Padro JA, Guardia E. Structure and dynamics of liquid ethanol, J Phys Chem. 1997; 101: 78-86.

[14] Skaf MS, Ladanyi BM. Molecular dynamics simulation of solvent dynamics in methanol-water mixtures, J Phys Chem. 1996; 100: 18258-18268.

[15] D' Angelo M, Onori G, Santucci A. Self association of monohydric alcohol in water: compressibility and infrared absorption measurements, J Chem Phys. 1994; 100: 3107-3113.

[16] Schwager F, Marand E, Davis RM. Determination of self-association equilibrium constants of ethanol by FTIR spectroscopy. J Phys Chem. 1996; 100: 19268-19272.

[17] Burikov S A, Dolenko T A, Kurchatov I S, Patsaeva S V, Starokurov Yu V. Computer analysis of vibrational spectra of aqueous ethanol solutions. Russian Phys J. 2012; 55: 383-388.

(c)) BY-NC-ND Licensed to GIRI

Support: authors declare that this study received no funding

Conflict of interest: authors declare there is no conflict of interest

Received: April 18 $8^{\text {th }}, 2014$; Revised: October $9^{\text {th }}, 2014$; Published: December $20^{\text {th }}, 2014$.

Correspondence author: Nirmal C. Sukul, West Bengal, India, ncsukul@gmail.com

How to cite this article: Chakraborty I, Datta S, Sukul A, Chakravarty R, Sukul NC. Variation in free and bound water molecules in different homeopathic potencies as revealed by their Fourier Transform Infrared Spectroscopy (FTIR). Int J High Dilution Res [online]. 2014 [cited YYYY Month dd]; 13(49): 189-196. Available from: http://www.feg.unesp.br/ ojs/index.php/ijhdr/article/view/716/738 